\section{Lumiracoxib provides effective pain relief for patients with knee osteoarthritis}

Nonsteroidal anti-inflammatory drugs are the preferred agents for treating chronic pain in knee osteoarthritis (OA), although they have known side effects of gastric irritation, renal impairment and reduced platelet aggregation. Selective cyclo-oxygenase 2 inhibitors (coxibs) might offer equivalent analgesia with a favorable safety profile. The novel coxib LUMIRACOXIB reaches higher concentrations in synovial fluid than in plasma; researchers, therefore, compared its short-term analgesic effects in patients with knee OA with those of placebo and celecoxib (the latter has known analgesic efficacy in knee OA).

Participants from 32 German centers were enrolled in this randomized, controlled trial. After an analgesic-free washout period, patients were given celecoxib ( $200 \mathrm{mg}$ twice daily, $n=145$ ), lumiracoxib (400 mg once daily, $n=144$ ) or placebo $(n=75)$. Pain scores were assessed at baseline and after 7 days of treatment. Despite a large placebo effect, as is typical of studies that assess pain scores, lumiracoxib provided superior analgesic efficacy to placebo.

Lumiracoxib provided rapid and effective analgesia, comparable with that of celecoxib. Pain relief was evident as early as $3 \mathrm{~h}$ after the first dose. The efficacy of analgesia was maintained between doses throughout the study, supporting the once-daily regimen of lumiracoxib used. The authors suggest that the rapid onset of analgesia seen with lumiracoxib is likely to benefit patients with knee $\mathrm{OA}$, who tend to need intermittent, rather than continuous, pain relief.

\section{Caroline Barranco}

Original article Wittenberg RH et al. (2006) First-dose analgesic effect of the cyclo-oxygenase- 2 selective inhibitor lumiracoxib in osteoarthritis of the knee: a randomized, double-blind, placebo-controlled comparison with celecoxib (NCT00267215). Arthritis Res Ther 8: R35

\section{Doxycycline plus methotrexate is more effective than methotrexate alone in early RA}

In addition to having antibacterial and immunomodulatory effects, tetracyclines have been shown to inhibit matrix metalloproteinases, which are responsible for connective-tissue breakdown in rheumatoid arthritis (RA). Minocycline is effective in the treatment of $\mathrm{RA}$, but has been associated with adverse effects including hyperpigmentation and dizziness; therefore, O'Dell et al. assessed the effectiveness of another tetracycline, doxycycline, in the treatment of early RA. In an attempt to determine whether antibacterial effects contributed to the mode of action of doxycycline in RA, twice-daily $100 \mathrm{mg}$ and $20 \mathrm{mg}$ doses were evaluated.

In all, 66 patients, all with a disease duration $<1$ year, were randomly allocated to receive methotrexate, plus either doxycycline or placebo. The primary endpoint was an ACR50 response after 2 years; this was achieved by $10 / 24(42 \%)$ in the $100 \mathrm{mg}$ doxycycline group, $7 / 18(39 \%)$ in the $20 \mathrm{mg}$ doxycycline group, and $3 / 24(13 \%)$ in the methotrexate-alone group. Patients who received doxycycline had greater improvements in each component of the ACR symptom questionnaire over 2 years than those who received methotrexate alone.

The similarity in efficacy of the two doxycycline doses favors the anti-metalloproteinase hypothesis for the mode of action of tetracyclines in RA. Two cases of potential photosensitivity rash were recorded in the high-dose doxycycline group, indicating a possible increase in the severity of these reactions when doxycycline is combined with methotrexate.

The authors conclude that doxycycline in combination with methotrexate is more effective than methotrexate alone for treatment of early RA.

\section{Jim Casey}

Original article O'Dell JR et al. (2006) Treatment of early seropositive rheumatoid arthritis: doxycycline plus methotrexate versus methotrexate alone. Arthritis Rheum 54: 621-627

\section{Cost-benefit analysis of tumor necrosis factor inhibitors in ankylosing spondylitis}

Tumor necrosis factor (TNF) inhibitors have beneficial effects when treating patients with ankylosing spondylitis (AS); however, the drug costs are high, and, therefore, a cost-benefit analysis could help to identify the best way to use these drugs. Boonen and colleagues constructed a Markov model to estimate the incremental costs and benefits of the anti-TNF agents etanercept and infliximab over 5 years,

\section{GLOSSARY}

LUMIRACOXIB

A cyclo-oxygenase 2

inhibitor that contains

a carboxylic acid group

instead of a sulfur group; it

reaches maximum plasma

concentration in $2 \mathrm{~h}$, has a

short plasma half-life of $\sim 4 \mathrm{~h}$

and does not accumulate in

the body

ACR50

A $50 \%$ improvement in symptoms according to the American College of Rheumatology criteria 